\title{
Modifying law enforcement training simulators for use in basic research
}

\author{
GUY O. SEYMOUR \\ Center of Excellence for Research on Training, Atlanta, Georgia \\ JEANNE M. STAHL \\ Center of Excellence for Research on Training \\ and Morris Brown College, Atlanta, Georgia \\ STEPHEN L. LEVINE \\ Human Touch Computer Consultants, Atlanta, Georgia \\ JOYCE L. INGRAM \\ Center of Excellence for Research on Training \\ and Georgia School of Professional Psychology, Atlanta, Georgia \\ and \\ R. FRED SMITH \\ Audio Intelligence Devices, Atlanta, Georgia
}

\begin{abstract}
Several approaches to using simulation as a method for teaching good judgment in the use of firearms by law enforcement officers and military personnel were investigated for their potential applications to research: the GAMMA Live Fire Video Training System, the FireArms Training Systems Simulator (FATS), and the Judgment Under Stress Training (JUST) simulator. Each instrument uses real-time, computer-controlled, life-sized projected video images of scenarios that may or may not present a threat, and handguns that fire real or simulated ammunition. The subjects respond to a sequence of "shoot/no-shoot" judgment scenarios resulting in data on judgment, reaction time, and accuracy. The JUST simulator was found to be more easily modifiable in capturing data on performance at microanalytic levels. Although true interactive simulations are not feasible with this system, this was not seen as a hindrance to its usefulness for research.
\end{abstract}

Law enforcement and military personnel need to be trained to employ good judgment in the use of firearms under situations of threat. Shoot/no-shoot computercontrolled simulators have been developed for this purpose. Such simulators have the potential to be used to acquire research data that allow evaluation of various factors that may influence the development and maintenance of good judgment. In this paper, we examine the efficacy for research of three state-of-the-art training systems and methodologies that use computer-controlled interactive hardware.

This research was supported in part by a grant from the United States Army to the first two authors. Correspondence concerning this article should be sent to G. O. Seymour, Center of Excellence for Research on Training, Morris Brown College, Atlanta, GA 30314. The following people can be contacted for information on the equipment: FATSBill Davis, Law Enforcement Sales, Firearms Training Systems, Inc., 110 Technology Parkway, Norcross, GA 30092, (404) 448-7318, FAX (404) 242-6962. GAMMA Live Fire Video Training System-Brian L. Danielson, Vice President of Marketing, Caswell International Corporation, 1221 Marshall Street N.E., Minneapolis, MN 55413-1035, (612) 379-2000, (612) 379-2367. JUST System-Donna Eagle, Vice President of Sales, Audio Intelligence Devices: 1-800-243-4642. Audio Intelligence Devices is a wholly owned subsidiary of Westinghouse, Inc.

\section{THE THREE SIMULATORS}

The Caswell International Live Fire Video Training System (GAMMA), the FireArms Training Systems (FATS), and the Judgment Under Stress Training (JUST) simulators were investigated. These instruments use real-time, computer-controlled, life-sized, projected video images of scenarios that may or may not present a threat. All the units are designed with a compact trainer's console that houses the computer, electronics, video equipment, and a printer. The units can be equipped with a camera and VCR system to allow replay of the action as feedback to the subject and a monitor for observation from another room. In addition, microphones can be used to give instructions during training and to record instructor and trainee verbalizations during testing. Actual firearms are used, either with real ammunition or with a laser or infrared-light-emitting device; this is modified to prevent accidental firing of live ammunition and includes "shot sound" hardware. The addition of speakers that produce "surround sound" enhances the effects. For realism, the laser and infraredlight systems have been developed with the balance, weight, and feel of firearms loaded with live ammunition. 
In addition, most FATS firearms allow the use of blanks, providing recoil and shell ejection.

The FATS and JUST systems are portable and can be used on most large movie screens, or even a blank wall. Because the GAMMA system uses live ammunition and requires a bullet trap, it is not as easily transported. Each system can be adapted to both individual and squad training for police SWAT teams and infantry. The subjects respond to a sequence of "shoot/no-shoot" judgment scenarios, resulting in data on judgment, reaction time, and accuracy.

All three systems present considerable improvements over the paper targets used in most shooting alleys, and they prevent the development of a form of "tunnel vision" that may develop from such training. Instead, trainees practice surveying the entire scene as it unfolds prior to making a judgment of whether or not to shoot. Reality is enhanced by requiring that trainees treat the scenarios as real situations as they unfold before them, using verbal commands or taking cover as appropriate.

The FATS, JUST, and GAMMA systems were designed as firearms judgment training equipment for military and law enforcement personnel-not for research. An examination of these systems determined that, with extensive modifications, any one of the systems could serve as an adequate research instrument. However, major differences were identified in their technological sophistication, adaptability to the requirements of research, and expense of software and hardware modifications necessary to access and manipulate data.

\section{The GAMMA Live Fire Video Training System}

This system was developed by Caswell International Corporation, a company that has been producing firing range, running man, and pop-up target systems since 1926. The GAMMA Live Fire Video Training System was developed to enhance accuracy training by presenting a more complex and realistic target. This allows for trainees to use their own individually sighted weapons and duty-issued ammunition in target practice without sacrificing the advantages of varied scenarios with contextual cues and distractors. This also cures the problem of dangerous tunnel vision that develops automatically with the use of paper-target ranges.

The GAMMA system technology uses life-sized videotaped images, presented with a high-resolution projection system on a screen consisting of several layers of paper on rollers. When the subject fires a round at the backlit video screen, a sensor attached to the screen causes the video to freeze, and then identifies the frame at which this occurred. The computerized database manager identifies the time by the number of frames between the presentation of the threat and the frame at which the screen sensor was activated. Subsequent bullets are similarly identified. This allows the system operator to give feedback to the trainees about the reaction time of their initial and subsequent responses. This sensor also triggers a bank of lights located in the bottom of the screen to illuminate the bullet holes. A video recorder collects the total image for later playback. The holes are then erased by covering them with a slight rolling of one of several sheets of paper.

The utility of the GAMMA system lies in its use of live ammunition as well as in the trainees' use of their own personally sighted weapons. However, its usefulness for research is limited by its inability to collect and store individualized and aggregate data other than that for immediate feedback to a particular trainee. This limitation was attenuated by the addition of a video recorder that filmed each trainee's performance with a wide-angle lens. However, because this camera is not linked directly to the GAMMA system, it can only function effectively as an ancillary and crude method for data collection.

\section{The FireArms Training System (FATS)}

The FATS simulator is the most technologically advanced and widely used small-arms training simulator for law enforcement and military personnel in the United States and abroad. The FATS simulator uses videotaped scenarios, converted to laser disk format, that are computer controlled in order to produce interactive "seamlessbranching" simulations. The subjects are presented with life-sized video scenarios that simulate situations of personal threat. They are provided with a laser-equipped firearm, and the videos are presented in a computer-controlled seamless-branching sequence in which a subject's inaction or action either raises or lowers, respectively, the level of threat presented on the video, without interrupting the action. The branching feature is especially attractive because realistic real-time consequences of shooting can be studied. For example, when a subject shoots the lethal area of the target, the target falls. When the subject misses, the target moves forward and "shoots" the subject in simulation of a real event. An added feature is that it allows an instructor to override the preset programmed branching alternatives in order to modify the outcome of a scenario on the basis of the actions of a trainee. The action of the subject creates "markers" that are then assessed, according to predetermined standards of performance, as to the accuracy of the shot fired and whether the action represented good or poor judgment. The option for the instructor to determine the adequacy of judgment and override the system is an additional positive feature of this system.

FATS semiautomatic firearms are provided with a simulated recoil action to ensure a realistic feel of the weapon during firing. In addition, shotguns, rifles, machine guns, and submachine guns with simulated action are available. Of the systems that do not use live ammunition, the $\mathrm{CO}_{2}$ operated recoil systems provide FATS with the greatest realism.

Two-lane, four-lane, and Squad Engagement Training Systems (SETS) are available. However, the squad training system, which can handle 13 laser-equipped weapons, 
was not a branching system at the time of this writing. Instead, feedback was provided by playing back the video with the "hits" marked on the screen.

The FATS simulator provides the instructor with a printed summary of the subject's performance. The major drawbacks to using the system for research are the inaccessibility of the raw data, the inability of the researcher to modify parameters (such as the point of threat), and the expense of converting customized videotapes to laser disk format and having them scored. The data produced by the off-the-shelf FATS simulator is averaged data. Access to the raw scores needed for research is unavailable without ordering a customized system. Scenarios can be scored prior to delivery according to the customer's special guidelines for use of force, but then they cannot be modified by the researcher. Thus, changes in research protocol would require returning the system to the company for modification. The modifications would then be fixed, and the initial settings could not be retrieved.

Although a large library of scenarios in several languages are available for the FATS, they primarily cover military and law enforcement situations. Our research requires customized scenarios, in which variables such as the gender, ethnicity, and age of the subjects in the scenario can be manipulated. In addition, when testing subjects who are not trained military or law enforcement personnel, there is a need for scenarios that present life situations outside of those well-defined operational parameters. Custom-made videos can be converted to laser disk format by FireArms Training Systems, Inc., but the cost of doing so is prohibitive. Of the systems using simulated ammunition, the superior technology of the FATS provides the most realistic and complete firearms training experience for the trainee. However, it is essentially a sealed system that cannot be modified by the user and, for these reasons, is not a good choice for research.

\section{The Judgment Under Stress Training (JUST) Simulator}

The JUST simulator was designed to offer the user the flexibility of a videotape system with which customized scenarios can be developed. It also preserves the immediacy of life-sized video presentations, for which judg- ment and accuracy can be measured individually and in aggregate. Because the JUST system presentation uses VHS videotapes-not laser disks-the outcome of the scenario cannot be modified while the scenario is being run. This is because branching capabilities, which are possible with video disks, are not yet possible even with highspeed videotape players. The JUST system does include a high-speed videotape player specially designed for this application. Its value lies in the ability to provide feedback to subjects immediately after their performance, based on the high-speed search capabilities of the system.

JUST uses real firearms that are modified to "shoot" an infrared-light beam that is recorded by the computer through an infrared-light-sensitive pinpoint camera for later playback. When giving feedback the scenario is replayed, and the coordinates showing the location at which the light gun hit the screen after it was fired are automatically marked by the system and displayed as crosshairs.

The advantages of the JUST system are that the researcher can access the raw data and modify any of the parameters, such as the point of the threat. In addition, the system was designed for the trainer who wishes to create customized scenarios at a relatively modest cost. Although we found that collection and storage of raw data required writing customized software, the expense was quite reasonable. Modifications of the equipment to expand its capabilities were easily accomplished, and they added gateways to link other psychological apparatuses for the simultaneous collection of data (e.g., pulse, heart rate, GSR monitors). These modifications were also available at a reasonable cost.

In the Simulator Laboratory at the Center of Excellence for Research on Training at Morris Brown College, we have acquired two projection systems that are designed to be linked to the JUST computer. One of these is a portable unit that allows the research laboratory to travel to where subject pools are usually engaged in training. Because the system can store group data, as in any database application, we are able to identify the session and date of both individual performances and group identifiers.

In summary, all three firearms judgment training simulators are suitable for research after considerable modifications to the manufacturer-provided system. 